\title{
Gamma Knife central lateral thalamotomy for the treatment of neuropathic pain
}

\author{
Andrea Franzini, MD, ${ }^{1}$ Luca Attuati, MD, ${ }^{1}$ Ismail Zaed, MD, ${ }^{1}$ Shayan Moosa, MD, ${ }^{2}$ \\ Antonella Stravato, $\mathrm{PhD},{ }^{3}$ Pierina Navarria, MD, ${ }^{3}$ and Piero Picozzi, MD ${ }^{1}$

\begin{abstract}
${ }^{1}$ Department of Neurosurgery, Humanitas Clinical and Research Center-IRCCS, Rozzano (Milano), Italy; ${ }^{2}$ Department of Neurological Surgery, University of Virginia Health Science Center, Charlottesville, Virginia; and ${ }^{3}$ Department of Radiation Oncology, Humanitas Clinical and Research Center-IRCCS, Rozzano (Milano), Italy
\end{abstract}

\begin{abstract}
OBJECTIVE The goal of this study was to assess the safety and efficacy of stereotactic central lateral thalamotomy with Gamma Knife radiosurgery in patients with neuropathic pain.

METHODS Clinical and radiosurgical data were prospectively collected and analyzed in patients with neuropathic pain who underwent Gamma Knife central lateral thalamotomy. The safety and efficacy of the lesioning procedure were evaluated by neurological examination and standardized scales for pain intensity and health-related quality of life. Visual analog scale (VAS) for pain, McGill Pain Questionnaire (MPQ), EuroQol-5 dimensions (EQ-5D), and the 36-Item Short Form Health Survey, version 2 (SF-36v2) were measured during baseline and postoperative follow-up evaluations at 3, $6,12,24$, and 36 months.
\end{abstract}

RESULTS Eight patients with neuropathic pain underwent Gamma Knife central lateral thalamotomy. Four patients suffered from trigeminal deafferentation pain, 2 from brachial plexus injury, 1 from central poststroke facial neuropathic pain, and 1 from postherpetic neuralgia. No lesioning-related adverse effect was recorded during the follow-up periods. All patients had pain reduction following thalamotomy. The mean follow-up time was 24 months. At the last follow-up visits, 5 patients reported $\geq 50 \%$ VAS pain reduction. The overall mean VAS pain score was 9.4 (range 8-10) before radiosurgery. After 1 year, the mean VAS pain score decreased significantly, from 9.4 (range 8-10) to 5.5 (mean $-41.33 \%$, $p=0.01$ ). $M P Q$ scores significantly decreased (mean $-22.18 \%, p=0.014$ ). Statistically significant improvements of the SF-36v2 quality of life survey (mean $+48.16 \%, p=0.012$ ) and $E Q-5 D(+45.16 \%, p=0.012$ ) were observed. At 2 years after radiosurgery, the VAS pain score remained significantly reduced to a mean value of $5.5(p=0.027)$. Statistically significant improvements were also observed for the MPQ (mean $-16.05 \%, p=0.034$ ); the $E Q-5 D$ (mean $+35.48 \%, p$ $=0.028$ ); and the SF-36v2 (mean $+35.84 \%, p=0.043$ ). At the last follow-up visits, pain had recurred in 2 patients, who were suffering from central poststroke neuropathic pain and brachial plexus injury, respectively.

CONCLUSIONS Safe, nonpharmacological therapies are imperative for the management of refectory chronic pain conditions. The present series demonstrates that Gamma Knife central lateral thalamotomy is safe and potentially effective in the long term for relieving chronic neuropathic pain refractory to pharmacotherapy and for restoring quality of life.

https://thejns.org/doi/abs/10.3171/2020.4.JNS20558

KEYWORDS thalamus; Gamma Knife; neuropathic pain; stereotactic surgery; stereotactic radiosurgery

$\mathrm{N}$ EUROPATHIC pain, defined by the International Association for the Study of Pain as "pain initiated or caused by a primary lesion or dysfunction of the nervous system," is a disabling disorder with limited effective treatments. Various surveys estimate the prevalence of chronic neuropathic pain to be approximately $10 \% .^{1,2}$ Chronic neuropathic pain is difficult to treat despite the use of multimodal therapies, with approximately $50 \%$ of patients achieving only partial relief. ${ }^{3}$

When pain persists in spite of conservative treatment, surgical therapies are then considered. Ablative brain surgery was widely used to treat medication-refractory chronic pain in the second half of the past century; however, this fell out of favor due to the introduction of intra-

ABBREVIATIONS CLp = posterior part of the central lateral nucleus; $\mathrm{Cm}=$ centromedian; $\mathrm{EQ}-5 \mathrm{D}=$ EuroQol-5 dimensions; $\mathrm{GKRS}=\mathrm{Gamma}$ Knife radiosurgery; $\mathrm{MPQ}=$ McGill Pain Questionnaire; MRgFUS = MR-guided focused ultrasound; Pf = parafascicular; RFTA = radiofrequency thermal ablation; SF-36v2 = 36-Item Short Form Health Survey, version 2; VAS = visual analog scale.

SUBMITTED February 23, 2020. ACCEPTED April 29, 2020.

INCLUDE WHEN CITING Published online July 24, 2020; DOI: 10.3171/2020.4.JNS20558. 
thecal opiate pumps and neuromodulative techniques in the 1990s. ${ }^{4}$ Recently, due to the development of advanced imaging techniques and safer devices for treatment delivery, interest in cerebral ablation has been rekindled. ${ }^{5,6}$

Thalamotomy, mesencephalotomy, and cingulotomy have been the most frequently performed ablative procedures for alleviating chronic pain. Notably, after the advent of stereotaxy, thalamotomy was performed more extensively because this procedure provided significantly less surgical and neurological morbidity than procedures performed at the pontine or mesencephalic level. ${ }^{7,8}$ At first, lesions were made in the lateral sensory-discriminative thalamus (ventrocaudal nucleus). ${ }^{8}$ However, due to an excessively high rate of somatosensory sequelae associated with these lesions, many surgeons went on to target the medial thalamic nuclei, which relieved pain without inducing any clinically detectable sensory loss or central pain. ${ }^{9,10}$ This type of pain relief is thought to occur because the medial thalamic nuclei have been shown to process predominantly the affective-motivational rather than the sensory-discriminative dimension of chronic pain. Within the thalamus, the posterior complex and the posterior part of the central lateral nucleus (CLp) are considered to have an intermediate functional role between the medial diffuse and lateral specific sensory nuclei and to project to large cortical domains, including areas mediating discriminative, affective-motivational, cognitive, and motor aspects of pain. Thus, lesioning of these areas is thought to have a multimodal effect. ${ }^{9}$ Some studies in which CLp thalamotomy was performed using radiofrequency thermal ablation (RFTA) ${ }^{9}$ or, more recently, MR-guided focused ultrasound (MRgFUS) have reported success in reducing pain as well as in reversing altered neurophysiological cortical activity related to chronic neuropathic pain states. ${ }^{11-14}$

In this study we investigated the safety and efficacy of stereotactic ablation of the thalamic CLp with Gamma Knife radiosurgery (GKRS) in a series of 8 patients suffering from neuropathic pain of nonmalignant origin.

\section{Methods \\ Data Collection}

Clinical and radiosurgical data were prospectively collected and analyzed for all patients who underwent GKRS ablation of the thalamic CLp for the treatment of chronic neuropathic pain refractory to conservative measures at our institution between 2015 and 2017. All patients had 12 to 36 months of follow-up after lesioning of the CLp using GKRS. Preoperative and postinterventional pain intensities and health-related quality of life measures were assessed using standardized scales (the visual analog scale [VAS] for pain in the range of $0-10$ [0, no pain; 10 , maximal pain]; the McGill Pain Questionnaire [MPQ]; ${ }^{15}$ the 36-Item Short Form Health Survey, version 2 [SF-36v2]; ${ }^{16}$ and the EuroQol-5 dimensions [EQ-5D] scale ${ }^{17}$ ). Pain and health-related quality of life measures were assessed before surgery and during follow-up visits.

\section{Target Selection}

After anterior and posterior commissure delineation on T2-weighted MR sequences, the target point was local- ized on 3D T1-weighted MR images at the following coordinates: 7 to $12 \mathrm{~mm}$ lateral (x), depending on the width of the third ventricle; 1 to $2 \mathrm{~mm}$ anterior to the posterior commissure (y); and 4 to $6 \mathrm{~mm}$ above the level of the intercommissural plane (z). In the stereotactic multiarchitectonic Morel atlas of the human thalamus and basal ganglia, these points correspond to the CLp. The interindividual variability of CLp is relatively low, in particular in the anteroposterior axis. This small variation can be easily taken into account by MRI visualization of neighboring landmark structures such as the posterior commissure, the habenula, and the stria medullaris. ${ }^{9}$

\section{Radiosurgical Treatment}

A Leksell G stereotactic coordinate frame (Elekta Instruments $\mathrm{AB}$ ) was attached to the patient's head and high-resolution MR images were obtained using 1-mmthick slices in the axial and coronal planes. Gadoliniumenhanced and nonenhanced T1-weighted and T2-weighted sequences were then acquired. The T1-weighted images were used for target localization. The coordinates of the target were entered into the planning software of the GKRS system (GammaPlan; Elekta Instruments AB). Radiosurgery was then performed with the Leksell Gamma Knife Perfexion system (Elekta Instruments AB). Singleisocenter dose planning was performed using a commercially available software system (GammaPlan). The maximum treatment dose with a single 4-mm collimator was determined jointly by the attending neurosurgeon, radiation oncologist, and medical physicist. The selection of the dose was guided by a dose-volume analysis based on prior experience with parenchymal tolerance to GKRS ${ }^{18-20}$ and on earlier reports of other authors investigating GKRS medial thalamotomy in the treatment of chronic pain., 421,22 The maximal dose delivered to the target ranged from 130 to $140 \mathrm{~Gy}$.

\section{Statistical Analysis}

Preoperative and postoperative scores were compared using the Wilcoxon signed-rank test, with $\mathrm{p}<0.05$ taken as statistically significant. The time points for the postoperative data used in the analysis were at 12 and 24 months. All analyses were performed with Stata 15 software (StataCorp LLC).

\section{Results}

Eight patients (50\% female) with neuropathic pain were treated with Gamma Knife central lateral thalamotomy. The mean age of the patients at surgery was 63.5 years (range 43-83 years). The patients suffered from trigeminal deafferentation pain due to multiple ablative procedures or surgical sectioning of the nerve and its ganglion in 4 cases, postherpetic neuralgia in 1 case, central poststroke neuropathic facial pain in 1 case, and neuropathic pain following brachial plexus injury in 2 cases. Four patients reported their pain as thermal (e.g., burning); 2 reported it as exteroceptive (e.g., pins and needles sensation); 1 reported it as proprioceptive (e.g., compressive and tearing sensation); and 1 reported it as electrical discharges. Pain and patient characteristics are shown in Table 1. The over- 


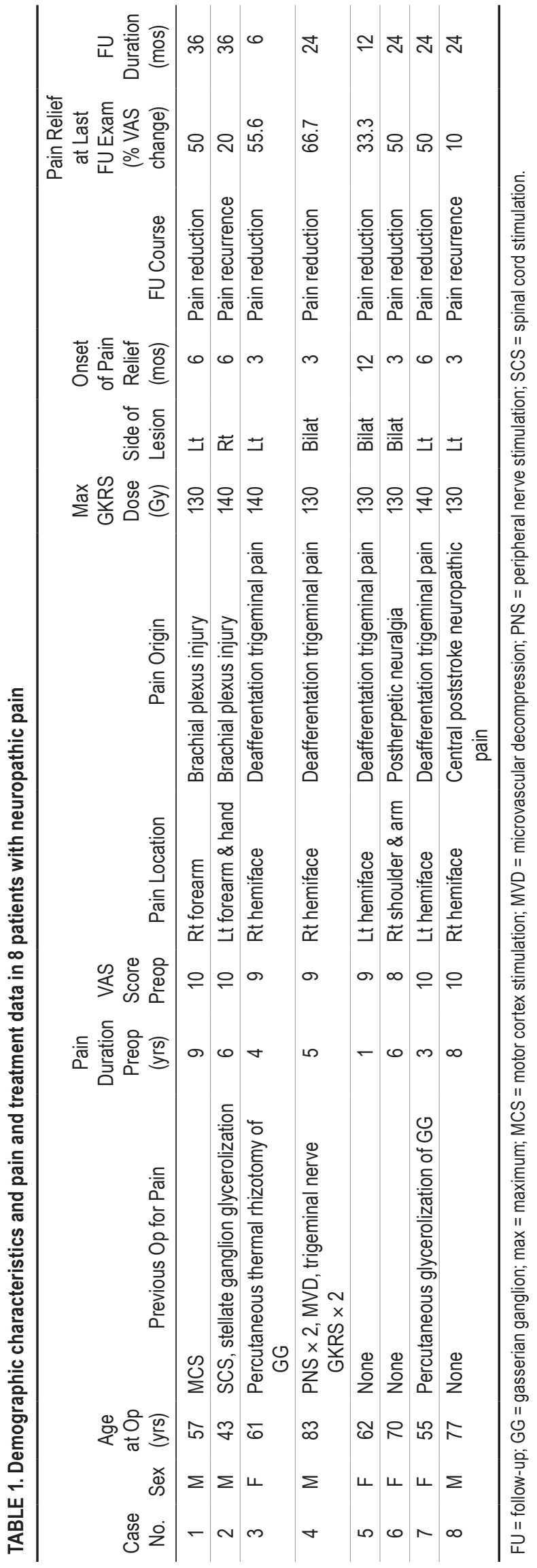


TABLE 2. Stereotactic coordinates of the radiosurgical lesions related to the commissural system in 8 patients with neuropathic pain

\begin{tabular}{|c|c|c|c|c|}
\hline \multirow{2}{*}{$\begin{array}{c}\text { Case } \\
\text { No. }\end{array}$} & \multirow{2}{*}{$\begin{array}{l}\text { Side of } \\
\text { Lesion }\end{array}$} & \multicolumn{3}{|c|}{ Stereotactic Coordinates of the Lesion Center $(\mathrm{mm})$} \\
\hline & & Lateral & Anteroposterior* & Vertical† \\
\hline 1 & Lt & 10 & 0 & +6.3 \\
\hline 2 & Rt & 8.4 & +1 & +6 \\
\hline 3 & $\mathrm{Lt}$ & 7 & +1 & +6.3 \\
\hline \multirow[t]{2}{*}{4} & $\mathrm{Rt}$ & 12 & +2 & +4 \\
\hline & $\mathrm{Lt}$ & 12 & +2 & +4 \\
\hline \multirow[t]{2}{*}{5} & Rt & 7 & +1 & +6 \\
\hline & $\mathrm{Lt}$ & 7 & +1 & +6 \\
\hline \multirow[t]{2}{*}{6} & Rt & 8 & +1 & +5 \\
\hline & Lt & 7 & +1 & +5 \\
\hline 7 & $\mathrm{Lt}$ & 7 & +1 & +6.3 \\
\hline 8 & $\mathrm{Lt}$ & 11 & +2 & +6.3 \\
\hline
\end{tabular}

* Values refer to + anterior to the posterior commissure.

† Values refer to + anterior and superior to the midcommissural point.

all mean VAS score was 9.4 before surgery (range 8-10). The mean duration of chronic pain prior to treatment was 5.25 years (median 5.5 years, range $1-9$ years). Maximum medical treatment had been unsuccessful in all patients, and all patients gave written informed consent to undergo radiosurgery.

Radiosurgical lesions were performed contralateral to the painful side in 5 cases and bilaterally in 3 cases. Stereotactic coordinates referring to the commissural system for each patient are listed in Table 2. There were no adjunctive signs or symptoms related to neurological damage, nor were there radiosurgery-related complications. In all patients the lesions were confirmed to be correctly placed at their intended targets on MR images that were acquired 6 months after the procedure (Figs. 1 and 2). The average follow-up time was 24 months in this study. The patients in cases 1 and 2 had follow-up at 3, 6, 12, 24, and 36 months; the patients in cases 3 and 5 had followup evaluations at 3, 6, and 12 months; and the patients in cases 4, 6, 7, and 8 had follow-up assessments at 3, 6, 12, and 24 months.
All patients attained pain reduction following GKRS. The mean latency to the clinical response was 5.5 months (median 4.5 months, range 3-12 months). After thalamotomy, 6 of 8 patients achieved 50\% VAS pain score reduction, from a mean value of 9.5 to 4.5 , within the 24 months of the follow-up period. The durations before reaching $50 \%$ reduction in VAS pain scores were between 6 and 24 months (median $17 \pm 8.0$ months). During the last followup (i.e., at 24 or 36 months), 5 of 8 patients reported $50 \%$ VAS pain score reduction. The overall mean VAS pain score was 9.4 (range 8-10) before radiosurgery. After 1 year the mean VAS pain score decreased significantly, from 9.4 (range 8-10) to 5.5 (mean $-41.33 \%, \mathrm{p}=0.01$ ). MPQ scores significantly decreased (mean $-22.18 \%, \mathrm{p}=$ $0.014)$. A statistically significant improvement of the SF$36 \mathrm{v} 2$ quality of life survey (mean $+48.16 \%, \mathrm{p}=0.012)$ and EQ-5D (+45.16\%, $\mathrm{p}=0.012)$ was also observed. Among the 6 patients with available follow-up data at the 2-year visit following radiosurgery, the VAS pain score was still significantly reduced to a mean value of $5.5(\mathrm{p}=0.027)$. Statistically significant improvements were also observed for the MPQ (mean $-16.05 \%, \mathrm{p}=0.034$ ); the EQ-5D (mean $+35.48 \%, \mathrm{p}=0.028)$; and the SF-36v2 (mean $+35.84 \%, \mathrm{p}$ $=0.043)($ Fig. 3).

At the last follow-up visits, pain had recurred in the patient in case 2 at the 24-month follow-up assessment and in the patient in case 8 at the 6-month follow-up assessment. The patient in case 8 suffered from central poststroke neuropathic pain, and the patient in case 2 suffered from brachial plexus injury.

\section{Discussion}

In this series of 8 patients with various therapy-resistant neuropathic pain syndromes, GKRS central lateral thalamotomy led to significant improvement in measures assessing the sensory and affective aspects of pain in all patients with an acceptable safety profile. In particular, this outcome proved long-lasting in most patients -6 of the 8 patients reported pain reduction from baseline after a mean follow-up period of 2 years. These results are noteworthy because medial thalamotomy for the treatment of neuropathic pain has not always been reliably effective and durable. ${ }^{9,10,19,23}$
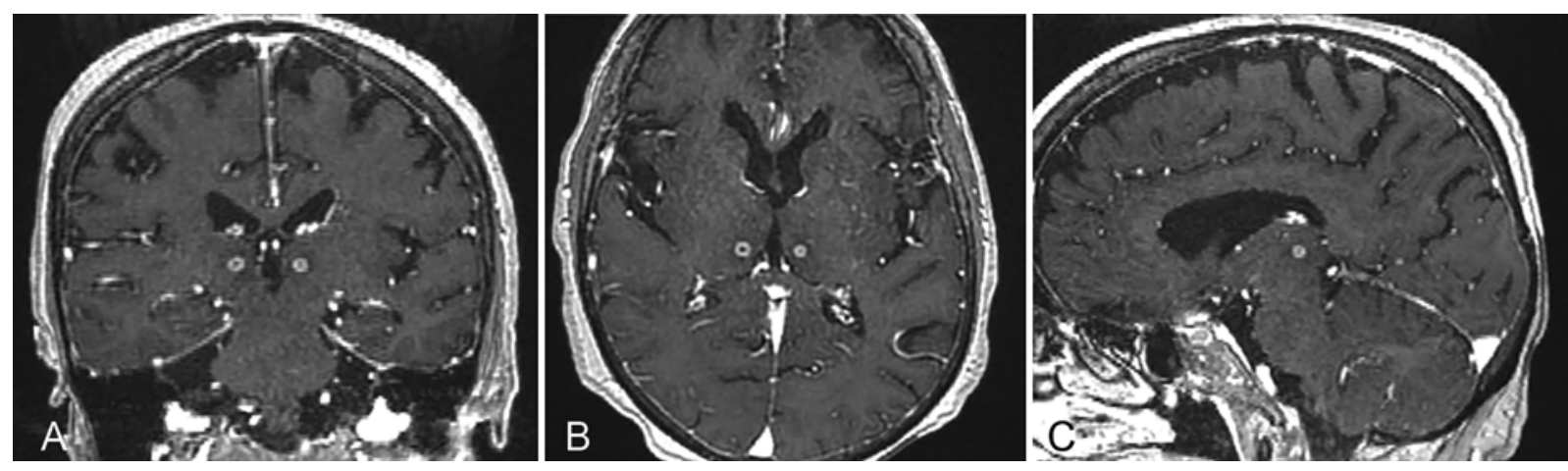

FIG. 1. Case 8. Coronal (A), axial (B), and sagittal (C) T1-weighted postcontrast MR images demonstrating a ring-enhancing lesion in the CLp of the thalamus. These images were acquired 6 months after radiosurgery. 

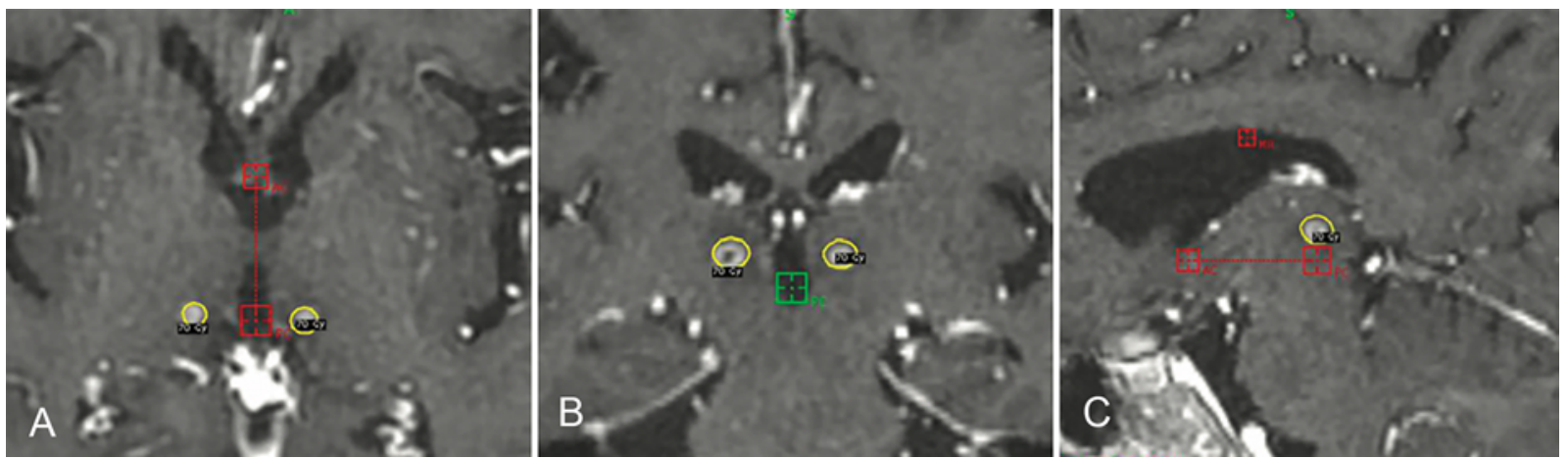

FIG. 2. Case 8 . The prescribed $50 \%$ isodose lines have been superimposed on the 6 -month postoperative T1-weighted postcontrast MR images acquired in the coronal (A), axial (B), and sagittal (C) planes. The exact correspondence between the prescribed isodose lines and the actual lesions demonstrates the high accuracy and precision of the GKRS lesioning technique. Figure is available in color online only.
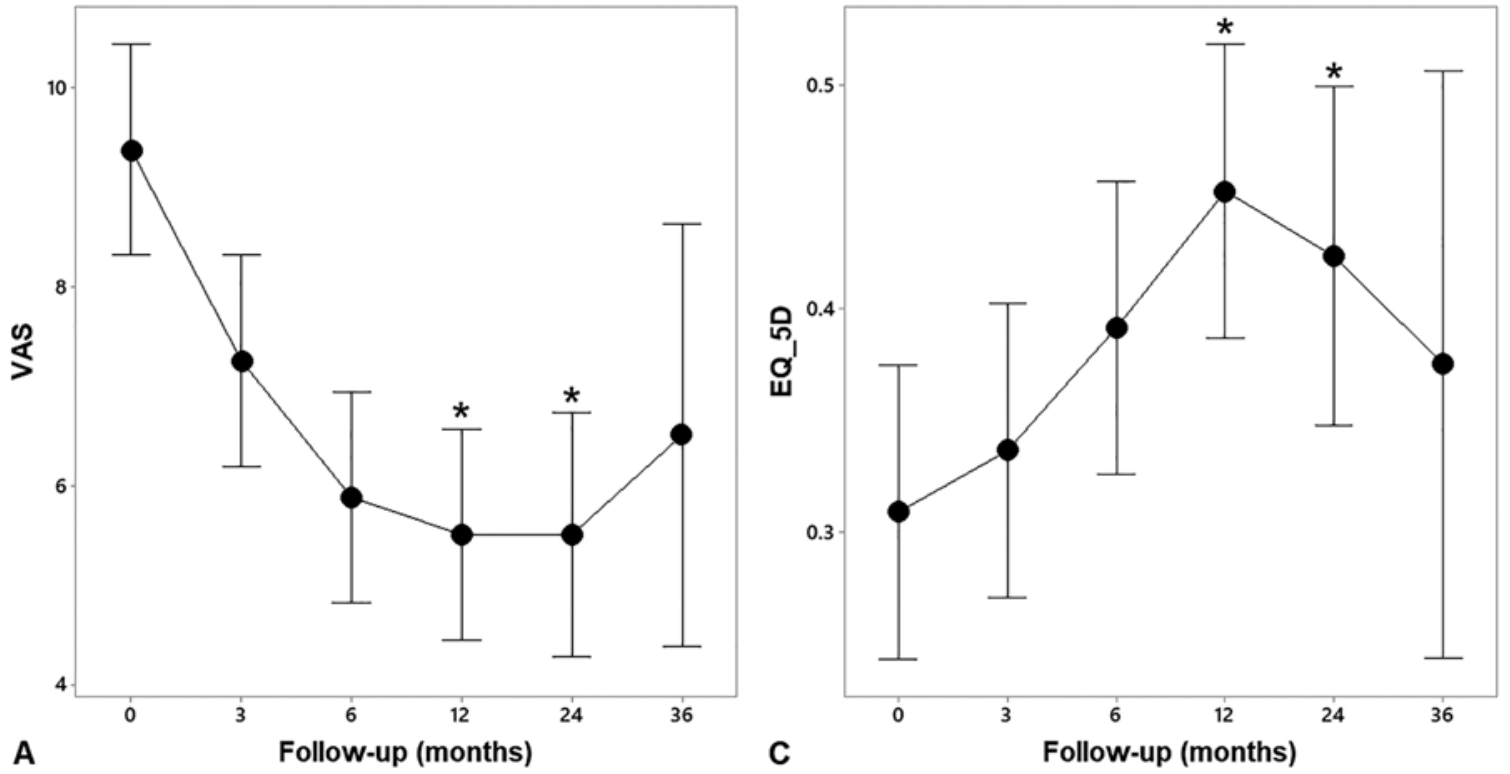

A Follow-up (months)

C
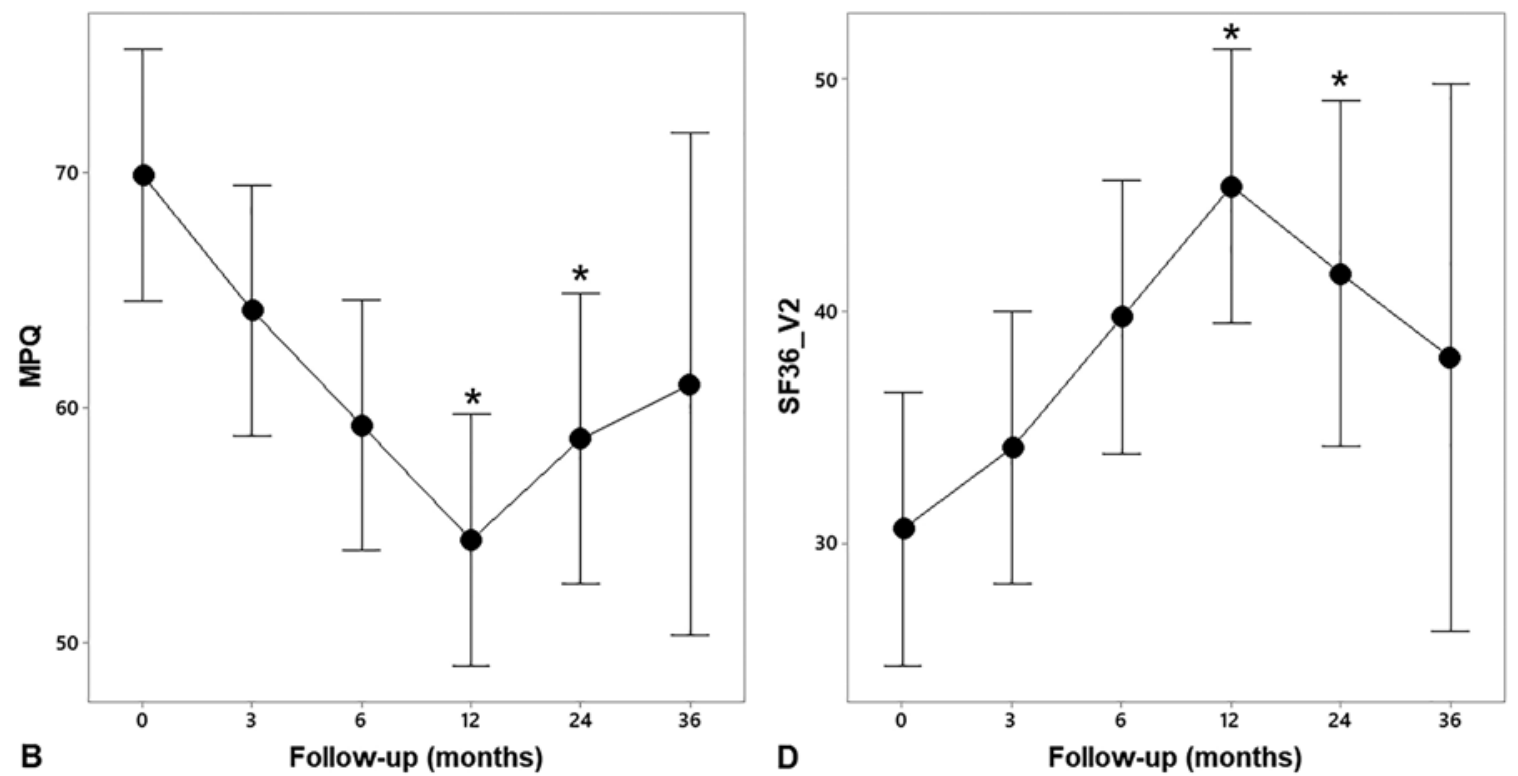

FIG. 3. Graphs showing patient outcomes after central lateral thalamotomy. A: VAS. B: MPQ. C: EQ-5D. D: SF-36v2. ${ }^{*} p<0.05$. 


\section{Mechanistic Effects of Central Lateral Thalamotomy}

Central lateral thalamotomy is a medial thalamotomy centered on the CLp. This thalamic area is considered to be a regulator of thalamocortical activity. The CLp is known to become dysfunctional in neuropathic pain syndromes; as such, it sustains and amplifies the production of low-frequency neurophysiological activity in thalamic and cortical locations. This increased production of theta rhythms within the CLp and cerebral cortex with high coherence between these 2 sites, which is known as thalamocortical dysrhythmia, is thought to be responsible for neurogenic pain and has been associated with chronic neuropathic pain states, as shown by several neurophysiological studies. ${ }^{9,24,25} \mathrm{CLp}$ ablation thus results in a resumption of normal thalamocortical dynamics and subsequent pain amelioration, without compromise of hemispheric functions, including nociception and cognition, and with no risk of causing deafferentation iatrogenic pain due to lesioning of the sensory-specific pain system. ${ }^{9}$

We based our target choice on the results of the works of Jeanmonod and colleagues. ${ }^{9,12}$ These authors reported favorable outcomes with CLp ablation for relieving chronic neuropathic pain, and, notably, their studies were conducted in a large series of patients who were followed for a long period of time.

\section{Outcomes of Medial Thalamotomy Studies}

There have been several reports of lesioning of the medial thalamus for the treatment of chronic pain. ${ }^{10,21-23,26-32}$ The most common thalamic targets have been the centromedian (CM) and parafascicular (Pf) nuclei, among other intralaminar nuclei. ${ }^{30}$ However, most studies with RFTA were conducted before modern imaging and physiological techniques were available, and therefore the actual lesion sites used for most series are uncertain. ${ }^{10}$ Results were generally worse and recurrence rates higher in patients with neuropathic pain. ${ }^{10,23,26} \mathrm{~A}$ review of the literature by Tasker identified 47 patients who underwent medial thalamotomy for the treatment of central and deafferentation pain, $29 \%$ of whom experienced pain relief (of note, some of these patients had additional lesions of other structures). ${ }^{10,23}$

GKRS for lesioning the medial thalamus was introduced by Leksell..$^{33}$ In 1980, Steiner et al. described the outcomes of lesioning the CM/Pf nuclei in 52 patients with cancer pain. In spite of the short survival of most patients included, pain reduction was not durable and was considered good in only 8 patients ( 3 of whom had pain recurrence within 1 year). ${ }^{22}$ Two decades later, Young and colleagues treated 61 patients with various chronic neuropathic and nociceptive pain syndromes. After a median follow-up period of 74 months, 32 patients achieved a worthwhile pain reduction. The target was the lateral portion of the medial dorsal nucleus, the intralaminar zone, and the $\mathrm{CM} / \mathrm{Pf}$ nuclei. However, high radiation doses were applied (140-180 Gy) and 1-3 isocenters were used. One patient died due to radionecrosis following bilateral thalamotomy performed using a high dose of radiation. . $^{31,32,34}$ These results were later confirmed in 2018 by Urgosik and Liscak, who reported a pain relief success rate in 13 of 30 (43\%) patients undergoing GKRS thalamotomy centered in the $\mathrm{CM} / \mathrm{Pf}$ nuclei. The median follow-up duration was 24 months, and no treatment complication was noticed. ${ }^{21}$
Finally, most recently, Lovo et al. also reported successful pain reduction in 6 of 10 patients treated with radiosurgical lesioning of the $\mathrm{CM} / \mathrm{Pf}$ nuclei. ${ }^{28} \mathrm{In}$ regard to lesioning the central lateral nucleus, Jeanmonod et al., in their most updated patient series of CLp thalamotomy performed using RFTA, reported that $53 \%$ of their patients continued to have $50 \%-100 \%$ pain reduction from baseline after an average follow-up period of 3 years and 9 months. Although more limited in follow-up duration, the $75 \%$ of patients who underwent CLp thalamotomy performed using MRgFUS by the same group also experienced considerable pain reduction..$^{12}$ The positive effects achieved by Jeanmonod et al. had been anticipated by some authors. As a matter of fact, Sano concentrated his efforts on the posterior part of the medial thalamus using a posterior approach, thus coming closer than anyone to targeting the CLp. ${ }^{30}$ Similarly, Hitchcock and Teixeira ${ }^{27}$ as well as Young et al. may have achieved good pain reduction for their patients because of the relatively large stereotactic lesions that they created, which included the posterior part of the CM/Pf complex that is quite close to the CLp..$^{10,32}$

The outcomes of Jeanmonod et al. from CLp lesioning are comparable with our own results, and they are generally favorable if compared to those of most authors who ablated various other nuclei of the medial thalamus for the treatment of neuropathic pain..$^{10,19}$ Jeanmonod et al. also reported an improvement in sensory function for some patients who previously experienced sensory dysfunction. Of note, we did not observe such an improvement, probably due to the latency interval of the clinical response to GKRS, which reduces the capacity for patients to detect slowly ongoing changes.

Initially, Jeanmonod et al. created thermal lesions in the CLp by using radiofrequency energy delivered through an interstitial rigid electrode ${ }^{9}$ and, subsequently, with transcranial high-intensity focused ultrasound. ${ }^{13,35}$ In both cases, the ablative procedure was guided by MRI. To our knowledge, no other groups have ever reported using central lateral thalamotomy for neuropathic pain with MR-guided radiosurgery. This is thus the first report ever investigating CLp thalamotomy with radiosurgery for neuropathic pain.

In most patients we performed unilateral CLp thalamotomy contralateral to the patient's most painful side, whereas Jeanmonod et al. mostly performed the lesions in a bilateral fashion. ${ }^{12,25}$ The rationale for bilateral CLp lesions reported by these authors is that the bilateral spinothalamic tracts project to the CLp (particularly significant for axial, face, and lower-extremity inputs) and that painrelated electroencephalographic overactivity is seen bilaterally. ${ }^{36}$ Our study, although limited by the small number of patients, suggests, however, that a unilateral lesion may suffice for achieving long-lasting pain reduction in some patients.

Supporting our findings, Steiner et al. proposed that unilateral lesions of the medial thalamic nuclei (both contralateral and ipsilateral to the patient's painful side) could lead to pain reduction. Interestingly, these authors also noted that, in their series of patients treated with centromedian thalamotomy, the effect on pain was usually more stable and of longer duration if the unilateral lesion was placed opposite to the painful side. ${ }^{22}$ Also, most other au- 
thors created thalamic lesions on the side contralateral to the patient's pain. Further data are necessary to determine if bilateral lesions result in more durable pain reduction than a unilateral procedure. However, bilateral lesions with GKRS should be used with caution due to the risk of adverse radiation effects. ${ }^{37} \mathrm{~A}$ safe radiation dose of 130 Gy with a single isocenter and 4-mm collimators and/or staging of the procedure have been safely used for bilateral thalamotomies in patients with movement disorders, and these may also be valid strategies to reduce the risk of adverse effects in patients with pain. ${ }^{38}$

In our study pain recurred in 2 patients, who were treated for central poststroke neuropathic pain and brachial plexus injury, respectively. Pain recurrence is common after stereotactic ablative procedures for chronic pain, including medial thalamotomy..$^{10}$ Interestingly, all 4 of the patients from our cohort who were treated for trigeminal deafferentation pain continued to have significant pain reduction at the last follow-up assessments. Likewise, the patients with trigeminal deafferentation pain described in the series of Lovo et al. had better results than those with other pain syndromes..$^{28}$ Although data are still limited, trigeminal deafferentation pain may respond better after central lateral thalamotomy than other neuropathic pain syndromes.

\section{Advantages and Drawbacks of Central Lateral Thalamotomy}

Aside from clinical efficacy, there are other reasons to target the CLp in patients suffering from chronic neuropathic pain. First, even in the acute postoperative phase, lesioning the CLp does not produce deficits in any neurological sphere, including somatosensory (pain sensation), motor, and neuropsychological (especially attention and state of consciousness) functions. ${ }^{9}$ Based on previous neurophysiological studies, this may be because the normal functioning of the CLp becomes transferred to other cerebral areas as a result of plastic reorganization from chronic pathology of the thalamocortical network. In contrast, ablative procedures that target the somatosensory pathways, including the ventrocaudal thalamic nucleus, carry the risk of producing deafferentation pain. Second, the location and large size of the CLp (the central lateral nucleus has a 4-mm diameter and is more than $12 \mathrm{~mm}$ in length) reduce the risk of producing damage to nearby eloquent areas, such as the pretectum and the lateral spinothalamic tract, even if a broad lesion is produced. Based on this, Jeanmonod's group and other authors who have performed ablations of the medial thalamus omitted physiological localization before creating the therapeutic lesion and concluded that it was unnecessary for these procedures. ${ }^{10}$ Central lateral thalamotomy can therefore be carried out safely with technologies such as GKRS that allow for only anatomical guidance.

Our results show that GKRS is a valid technique for central lateral thalamotomy. GKRS, like MRgFUS, has a greater accuracy than RFTA because there is no mechanical brain shift from the introduction of a rigid electrode into the intracranial space. ${ }^{12,39}$ Also, the trajectory of the needle electrode from a site anterior to the coronal suture to the CLp inevitably penetrates the ventricle and ependy- mal layer, ${ }^{9}$ which is a factor known to decrease procedural accuracy. ${ }^{39}$ Additionally, due to the transcranial nature of GKRS, the risk of surgery-related infection is virtually eliminated, as well as the risk of intracranial hemorrhage that may occur from an intracranial probe. Finally, GKRS has been shown to allow for better control of the extent of a lesion and subsequently to lower the interindividual variability of effect, especially when compared to RFTA. ${ }^{19,29,40}$ The size of GKRS brain functional lesions with doses of 130-140 Gy is similar to those created with MRgFUS, as long as a single isocenter shot with 4-mm collimators is used. Using both modalities, lesions appear on MRI as small and well-demarcated ring-enhancing areas of approximately 4- to 5-mm diameter. Without blocking or plugging the collimators' array, GKRS lesions are spheroid ${ }^{41}$ whereas MRgFUS lesions are slightly oval and elongated along the $\mathrm{z}$ axis. ${ }^{41,42}$ The main difference between the two treatment modalities is that full maturation to necrosis occurs 6-9 months after radiosurgery, whereas this process is complete within 48-72 hours following MRgFUS ${ }^{43}$ Human and animal studies have also shown that the borders of GKRS lesions are less distinct, reflecting the more gradual dose falloff of radiosurgery. ${ }^{41,43-45}$

Despite advantages, GKRS does have some drawbacks when compared to other techniques that have been used for thalamotomy. The latency interval for lesion generation delays the beneficial effect; thus, techniques such as RFTA and MRgFUS, which allow for an immediate effect, may be preferred in select cases in which a patient requires immediate pain relief. However, all of our patients were suffering from chronic pain states that had lasted for several years and did not necessitate an urgent treatment. Another possible concern is the occurrence of radiation-related adverse events, including radionecrosis and secondary malignancies, as well as the dose-accumulation effect of ionizing radiation. The latter may be relevant, because patients with chronic neuropathic pain have a high rate of pain recurrence following ablative brain surgery. The limited possibility of repeating GKRS due to radiation dose-accumulation effect would reduce the overall duration of pain control. Finally, the GKRS procedure lacks physiological guidance for lesion creation, which is possible with RFTA and MRgFUS. However, other authors have reported successful outcomes after lesioning the CLp or the medial thalamus without any physiological guidance and have considered it unnecessary for central lateral thalamotomy. ${ }^{12}$

\section{Limitations of the Study}

There are several limitations of this study, including the fact that it is a single-center experience. There are also a relatively small number of patients, which limits the power and generalizability of the study. In addition, no pre- or postoperative cognitive evaluations were performed for the patients included in this study. Further randomized prospective studies will have to be conducted in a larger series of patients to establish the true efficacy of GKRS central lateral thalamotomy in the treatment of patients with medication-refractory neuropathic pain. A longer follow-up period is also needed to determine pain recurrence rates and any late-stage effects of radiosurgery. 


\section{Conclusions}

The results of our study, which reports a preliminary experience, suggest that central lateral thalamotomy with GKRS is a safe and effective procedure for the treatment of patients with treatment-refractory neuropathic pain. We confirm the findings of the previously reported studies of Jeanmonod et al., to which our results are comparable. Of note, this is the first report investigating the efficacy of GKRS central lateral thalamotomy for chronic neuropathic pain. In our series, central lateral thalamotomy was effective in reducing various types of neuropathic pain; however, the number of patients in this study is too small to infer the role of this procedure in the management of distinct neuropathic pain syndromes. We observed that all patients suffering from deafferentation trigeminal pain had long-term pain reduction with no recurrence, thus suggesting that these patients are good candidates for central lateral thalamotomy. Further validation in a larger group of patients is warranted to make such conclusions.

\section{Acknowledgments}

This work was supported by Humanitas Research Hospital, Via Manzoni 56, Rozzano (Milano), Italy. We thank Chang-Chia Liu, PhD (a neuroscientist at the Human Brain Function Laboratory, University of Virginia Health Science Center), and Emanuela Morenghi for helping with statistical analyses.

\section{References}

1. Torrance N, Smith BH, Bennett MI, Lee AJ. The epidemiology of chronic pain of predominantly neuropathic origin. Results from a general population survey. J Pain. 2006;7(4): 281-289.

2. Yawn BP, Wollan PC, Weingarten TN, et al. The prevalence of neuropathic pain: clinical evaluation compared with screening tools in a community population. Pain Med. 2009; 10(3):586-593.

3. Dworkin RH, O'Connor AB, Backonja M, et al. Pharmacologic management of neuropathic pain: evidence-based recommendations. Pain. 2007;132(3):237-251.

4. Young RF, Jacques DS, Rand RW, Copcutt BR. Medial thalamotomy with the Leksell Gamma Knife for treatment of chronic pain. Acta Neurochir Suppl (Wien). 1994;62:105-110.

5. Franzini A, Moosa S, Servello D, et al. Ablative brain surgery: an overview. Int J Hyperthermia. 2019;36(2):64-80.

6. Franzini A, Moosa S, Prada F, Elias WJ. Ultrasound ablation in neurosurgery: current clinical applications and future perspectives. Neurosurgery. 2019; nyz407.

7. Laitinen LV. Mesencephalotomy and thalamotomy for chronic pain. In: Lunsford LD, ed. Modern Stereotactic Neurosurgery. Springer; 1988:269-277.

8. Hariz MI, Bergenheim AT. Thalamic stereotaxis for chronic pain: ablative lesion or stimulation? Stereotact Funct Neurosurg. 1995;64(1):47-55.

9. Jeanmonod D, Morel A. The central lateral thalamotomy for neuropathic pain. In: Lozano AM, Gildenberg PL, Tasaker RR, ed. Textbook of Stereotactic and Functional Neurosurgery. Springer; 2009:2081-2096.

10. Tasker RR. Stereotactic medial thalamotomy for chronic pain: is it an effective procedure. In: Burchiel KJ, ed. Surgical Management of Pain. Thieme; 2002:805-811.

11. Gallay MN, Moser D, Jeanmonod D. Safety and accuracy of incisionless transcranial MR-guided focused ultrasound functional neurosurgery: single-center experience with 253 targets in 180 treatments. J Neurosurg. 2018;130(4):12341243.

12. Jeanmonod D, Werner B, Morel A, et al. Transcranial magnetic resonance imaging-guided focused ultrasound: noninvasive central lateral thalamotomy for chronic neuropathic pain. Neurosurg Focus. 2012;32(1):E1.

13. Martin E, Jeanmonod D, Morel A, et al. High-intensity focused ultrasound for noninvasive functional neurosurgery. Ann Neurol. 2009;66(6):858-861.

14. Sarnthein J, Stern J, Aufenberg C, et al. Increased EEG power and slowed dominant frequency in patients with neurogenic pain. Brain. 2006;129(Pt 1):55-64.

15. Melzack R. The McGill Pain Questionnaire: major properties and scoring methods. Pain. 1975;1(3):277-299.

16. Lins L, Carvalho FM. SF-36 total score as a single measure of health-related quality of life: scoping review. SAGE Open Med. 2016;4:2050312116671725.

17. Brazier J, Jones N, Kind P. Testing the validity of the Euroqol and comparing it with the SF-36 health survey questionnaire. Qual Life Res. 1993;2(3):169-180.

18. Andersson B, Larsson B, Leksell L, et al. Histopathology of late local radiolesions in the goat brain. Acta Radiol Ther Phys Biol. 1970;9(5):385-394.

19. Kondziolka D, Flickinger JC, Lunsford LD. Stereotactic radiosurgery for epilepsy and functional disorders. Neurosurg Clin N Am. 2013;24(4):623-632.

20. Kondziolka D, Lunsford LD, Claassen D, et al. Radiobiology of radiosurgery: Part I. The normal rat brain model. Neurosurgery. 1992;31(2):271-279.

21. Urgosik D, Liscak R. Medial Gamma Knife thalamotomy for intractable pain. J Neurosurg. 2018;129(suppl 1):72-76.

22. Steiner L, Forster D, Leksell L, et al. Gammathalamotomy in intractable pain. Acta Neurochir (Wien). 1980;52(3-4): $173-184$

23. Tasker RR. Thalamotomy. Neurosurg Clin N Am. 1990;1(4): 841-864.

24. Llinás RR, Ribary U, Jeanmonod D, et al. Thalamocortical dysrhythmia: a neurological and neuropsychiatric syndrome characterized by magnetoencephalography. Proc Natl Acad Sci U S A. 1999;96(26):15222-15227.

25. Jeanmonod D, Schulman J, Ramirez R, et al. Neuropsychiatric thalamocortical dysrhythmia: surgical implications. Neurosurg Clin N Am. 2003;14(2):251-265.

26. Dougherty PM, Lee J-I, Dimitiou T, Lenz FA. Medial thalamotomy. In: Burchiel KJ, ed. Surgical Management of Pain. Thieme; 2002.

27. Hitchcock ER, Teixeira MJ. A comparison of results from center-median and basal thalamotomies for pain. Surg Neurol. 1981;15(5):341-351.

28. Lovo EE, Torres B, Campos F, et al. Stereotactic gamma ray radiosurgery to the centromedian and parafascicular complex of the thalamus for trigeminal neuralgia and other complex pain syndromes. Cureus. 2019;11(12):e6421.

29. Régis J. Gamma knife for functional diseases. Neurotherapeutics. 2014;11(3):583-592.

30. Sano K. Intralaminar thalamotomy (thalamolaminotomy) and postero-medial hypothalamotomy in the treatment of intractable pain. In: Pain-Its Neurosurgical Management. Vol. 8. Karger Publishers; 1977:50-103.

31. Young RF, Jacques DB, Mark R, Copcutt B. 794 Gamma Knife medial thalamotomy for treatment of chronic pain: long-term results. Neurosurgery. 2001;49(2):534.

32. Young RF, Vermeulen SS, Grimm P, et al. Gamma Knife thalamotomy for the treatment of persistent pain. Stereotact Funct Neurosurg. 1995;64(suppl 1):172-181.

33. Leksell L. Cerebral radiosurgery. I. Gammathalanotomy in two cases of intractable pain. Acta Chir Scand. 1968;134(8): 585-595.

34. Young RF, Jacques DS, Rand RW, et al. Technique of stereo- 
tactic medial thalamotomy with the Leksell Gamma Knife for treatment of chronic pain. Neurol Res. 1995;17(1):59-65.

35. Gallay MN, Moser D, Rossi F, et al. Incisionless transcranial MR-guided focused ultrasound in essential tremor: cerebellothalamic tractotomy. J Ther Ultrasound. 2016;4:5.

36. Michels L, Moazami-Goudarzi M, Jeanmonod D. Correlations between EEG and clinical outcome in chronic neuropathic pain: surgical effects and treatment resistance. Brain Imaging Behav. 2011;5(4):329-348.

37. Okun MS, Stover NP, Subramanian T, et al. Complications of gamma knife surgery for Parkinson disease. Arch Neurol. 2001;58(12):1995-2002.

38. Niranjan A, Raju SS, Monaco EA, et al. Is staged bilateral thalamic radiosurgery an option for otherwise surgically ineligible patients with medically refractory bilateral tremor? $J$ Neurosurg. 2018;128(2):617-626.

39. Holl EM, Petersen EA, Foltynie T, et al. Improving targeting in image-guided frame-based deep brain stimulation. Neurosurgery. 2010;67(2 Suppl Operative):437-447.

40. Hirabayashi H, Hariz MI, Wårdell K, Blomstedt P. Impact of parameters of radiofrequency coagulation on volume of stereotactic lesion in pallidotomy and thalamotomy. Stereotact Funct Neurosurg. 2012;90(5):307-315.

41. Lunsford LD, Altschuler EM, Flickinger JC, et al. In vivo biological effects of stereotactic radiosurgery: a primate model. Neurosurgery. 1990;27(3):373-382.

42. Wintermark M, Huss DS, Shah BB, et al. Thalamic connectivity in patients with essential tremor treated with MR imaging-guided focused ultrasound: in vivo fiber tracking by using diffusion-tensor MR imaging. Radiology. 2014;272(1): 202-209.

43. Elias WJ, Khaled M, Hilliard JD, et al. A magnetic resonance imaging, histological, and dose modeling comparison of focused ultrasound, radiofrequency, and Gamma Knife radiosurgery lesions in swine thalamus. J Neurosurg. 2013;119(2): 307-317.
44. Friehs GM, Norén G, Ohye C, et al. Lesion size following Gamma Knife treatment for functional disorders. Stereotact Funct Neurosurg. 1996;66(suppl 1):320-328.

45. Wintermark M, Druzgal J, Huss DS, et al. Imaging findings in MR imaging-guided focused ultrasound treatment for patients with essential tremor. AJNR Am J Neuroradiol. 2014; 35(5):891-896.

\section{Disclosures}

The authors report no conflict of interest concerning the materials or methods used in this study or the findings specified in this paper.

\section{Author Contributions}

Conception and design: Franzini, Picozzi. Acquisition of data: Attuati, Zaed, Stravato, Picozzi. Analysis and interpretation of data: Franzini, Picozzi. Drafting the article: Franzini, Moosa. Critically revising the article: Franzini, Attuati, Zaed, Moosa, Stravato, Picozzi. Reviewed submitted version of manuscript: Franzini, Zaed, Moosa, Stravato, Picozzi. Approved the final version of the manuscript on behalf of all authors: Franzini. Statistical analysis: Franzini. Administrative/technical/material support: Attuati, Stravato, Navarria, Picozzi. Study supervision: Picozzi.

\section{Correspondence}

Andrea Franzini: Humanitas Clinical and Research Center, IRCCS, Rozzano (Mi), Italy. andrea.franzini1@hotmail.it. 